Discrete Comput Geom 28:313-330 (2002)

DOI: $10.1007 / \mathrm{s} 00454-002-0694-2$

\title{
Isoperimetric Constants of Infinite Plane Graphs*
}

\author{
Serge Lawrencenko, ${ }^{1}$ Michael D. Plummer, ${ }^{2}$ and Xiaoya Zha ${ }^{3}$ \\ ${ }^{1}$ Department of Mathematics and Statistics, Rochester Institute of Technology, \\ Rochester, NY 14623, USA \\ ${ }^{2}$ Department of Mathematics, Vanderbilt University, \\ Nashville, TN 37240, USA \\ michael.d.plummer@vanderbilt.edu \\ ${ }^{3}$ Department of Mathematical Sciences, Middle Tennessee State University, \\ Murfreesboro, TN 37132, USA \\ xzha@.mtsu.edu
}

\begin{abstract}
Let $G$ be an infinite locally finite plane graph with one end and let $H$ be a finite plane subgraph of $G$. Denote by $a(H)$ the number of finite faces of $H$ and by $l(\partial H)$ the number of the edges of $H$ that are on the boundary of the infinite face or a finite face not in $H$. Define the isoperimetric constant $h(G)$ to be $\inf _{H} l(\partial H) / a(H)$ and define the isoperimetric constant $h(\delta)$ to be $\inf _{G} h(G)$ where the infimum is taken over all infinite locally finite plane graphs $G$ having minimum degree $\delta$ and exactly one end. We establish the following bounds on $h(\delta)$ for $\delta \geq 7$ :
\end{abstract}

$$
\frac{(\delta-6)\left(\delta^{2}-8 \delta+15\right)}{(\delta-4)\left(\delta^{2}-8 \delta+13\right)} \leq h(\delta) \leq \sqrt{\frac{\delta-6}{\delta-2}} .
$$

\section{Introduction}

The subject matter of this paper is hyperbolic infinite triangulations, and, generally, infinite cell 2-complexes $G$ with one end - that is, the carrier of $G$ is homeomorphic to the plane or, in other words, all points in the plane are covered by $G$. We draw parallels between the metric properties of the cell 2-complexes and their continuous counterparts. This approach gives rise to several discrete extremal problems. The purpose of this paper is to establish an upper bound and new lower bounds for the isoperimetric constants of $G$.

* The work of Xiaoya Zha was supported by NSF Grant DMS-9622780 and Middle Tennessee State University Faculty Research Grant (2000). 
Let $M^{2}$ be a topological 2-manifold. Let $G$ be a cell 2-complex homeomorphic to $M^{2}$ (more accurately, the carrier of $G$ is homeomorphic to $M^{2}$ ). It is a well known fact that if $M^{2}$ is noncompact (compact, respectively), then $G$ is infinite (finite). By $V(G), E(G)$, and $F(G)$ we denote the sets of the vertices (0-cells), edges (1-cells), and faces (2-cells) of $G$, respectively. Especially, $G$ is called a triangulation of $M^{2}$ when it is simplicial. The faces of a triangulation are also called triangles. The degree of $v \in V(G)$, denoted by $\operatorname{deg}_{G}(v)$, is the number of the edges of which $v$ is a vertex. The star of $v \in V(G)$, denoted by $\operatorname{st}(v)$, is the union of the cells of which $v$ is a vertex. $G$ is $\delta$-regular if each vertex of $G$ has degree $\delta$.

The following is a classical geometric fact which provides background and motivation for the present research. Let $M^{2}$ be a simply connected Riemannian 2-manifold which is complete as a metric space. If the Gaussian curvature $K$ is uniformly bounded above by a negative constant,

$$
K(p) \leq-\varepsilon^{2}<0
$$

for each point $p \in M^{2}$, then for any piecewise smooth closed curve $C$ bounding a 2disk $D$,

$$
\frac{\text { length } C}{\text { area } D} \geq \varepsilon \text {. }
$$

Letting $C$ vary and taking the infimum of these ratios leads to a positive number which is an important type of so-called isoperimetric constant of $M^{2}$ (see [C], [G], and [Y]), which we refer to as the Cheeger-Gromov-Yau constant.

Let $G$ be a cell 2-complex homeomorphic to a closed topological 2-manifold $M^{2}$. For a vertex $v \in V(G)$, the combinatorial curvature $K_{c}(v)$ is defined to be $2 \pi$ minus the sum of the angles around $v$, where each face of $G$ is thought of as a Euclidean regular polygon. Thus $G$ models a metric of $M^{2}$ by way of the combinatorial curvature $K_{c}$ which is a discrete analogue of the Gaussian curvature $K$. In this interpretation, if $G$ is $\delta$-regular, restriction on the minimum degree $\delta$ of $G$ becomes a restriction on the curvature of $M^{2}$. The larger $\delta$, the more curved (negatively) $M^{2}$ is. The hyperbolicity of $M^{2}$ is guaranteed by the condition $\delta \geq 7$. This condition is a discrete analogue of condition (1.1), while a 6-regular triangulation models a flat 2-manifold.

We deal with a specific situation in which $M^{2}$ is the plane and $G$ is a plane graph-that is, an embedding of a planar locally finite graph (1-complex) in the plane. Thus a plane graph is a two-dimensional object; it is determined by the triple of sets $\{V(G), E(G)$, $F(G)$ \}. We restrict our attention to the case in which $G$ has one end. This restriction, along with the condition $\delta \geq 7$ (or even $\delta \geq 6$ ), guarantees, by Corollary 6.4, that $G$ corresponds to a cell 2-complex in which every face is bounded by a cycle (no repeated vertices), and two faces may intersect only at a single vertex or a single edge. A plane cell 2-complex is one homeomorphic to the plane. It is necessarily infinite and locally finite [M2]. The case of a single end is of particular interest thanks to the fact that some infinite locally finite plane graphs with one end are the universal coverings of finite graphs on closed compact surfaces. Furthermore, it has turned out [PZ] that such plane graphs also have a structure similar to that of graph embeddings on surfaces with large representativity and minimum degree at least 6.

A finite plane subgraph of $G$ is a plane embedding of the graph determined by fixing finite subsets of $V(G)$ and $E(G)$, naturally inherited from $G$. 
Let $H$ be a finite plane subgraph of $G$. Denote by $a(H)$ the number of finite faces of $H$ (the "area" of $H$ ), and by $l(\partial H)$ the number of the edges of $H$ that are on the boundary of at most one finite face (the "perimeter" of $H$ ). By analogy with the continuous case, define the discrete isoperimetric constant $h(G)$ by

$$
h(G)=\inf _{H} \frac{l(\partial H)}{a(H)},
$$

and define the $\delta$-isoperimetric constant $h(\delta)$ by

$$
h(\delta)=\inf _{G} h(G),
$$

where the infimum is taken over all plane cell 2-complexes $G$ with minimum degree $\delta$. We show in Section 4 that we may assume that $G$ is a triangulation when estimating (1.4) and that $H$ is a polygon in $G$ when estimating (1.3), in which case $l(\partial H)=|E(\partial H)|$ and $a(H)=|F(H)|$, where $\partial H$ denotes the boundary of $H$. A (triangulated) polygon means a subcomplex of $G$, the carrier of which is a 2-disk, and a 2-disk means a topological space homeomorphic to the region determined by inequality $x^{2}+y^{2} \leq 1$ in the Euclidean $(x, y)$-plane. If $G$ is a cell 2-complex homeomorphic to a closed 2-manifold, then $\operatorname{st}(v)$ is a 2-disk for each $v \in V(G)$.

Remark. In the literature, two commonly used definitions of the isoperimetric constant of an arbitrary connected graph $G$ are

$$
i_{p}(G)=\inf _{X}\left\{\frac{|d(X)|}{m(X)}\right\}
$$

(see [M1], [W], [Do], [HS], and [Z]) and

$$
i(G)=\inf _{X}\left\{\frac{|d(X)|}{|X|}\right\}
$$

(see [HJL] and [M1]), where $d(X)$ denotes the set of edges having one end in $X$ and the other in $V(G)-X$ and $m(X)$ denotes the sum of the degrees of the vertices in $X$. In each case the infimum is taken over all nonempty finite sets of vertices $X \subseteq V(G)$. When graph $G$ is embedded in a surface, $h(G)$ and $i(G)$ are dual concepts and $h(G)=i\left(G^{*}\right)$ where $G^{*}$ is the surface dual of $G$. When $G$ is $k$-vertex-regular, then $i(G)=k i_{p}(G)$.

While the isoperimetric constants $i(G)$ and $i_{p}(G)$ are used in the study of the growth rate of graphs, random walks, difference Laplacians and percolation on graphs as well as some other problems, we prefer to use $h(G)$ here (also see [HS]) as the isoperimetric constant. It seems to us somewhat more "natural" to do so, because this definition captures more geometric meaning in the sense that it is a discrete analog of Cheeger's isoperimetric inequality $[\mathrm{C}]$ (which is the quotient of the measure of the $(n-1)$-dimensional geometric boundary of an $n$-dimensional submanifold and the measure of the submanifold itself; i.e., "area" over "volume").

In this paper we deal with the problem of estimating $h(G)$ and $h(\delta)$ for plane cell 2-complexes $G$ with $\delta \geq 7$. It can be easily seen that $h(G)=0$ if $G$ is a 6-regular plane 
triangulation. A number of people [Do], [BMST], [M1], [M3], [M4], [MW], [HJL], [HS], [Z], [S], [W] have looked at the isoperimetric constant $h(G)$, sometimes in the form of the plane dual. Here for the sake of uniformity and completeness, we restate some of these results in terms of $h(\delta)$. The first is a constant bound obtained by Dodziuk [Do]:

$$
h(\delta) \geq \frac{1}{26} \quad(\delta \geq 7) .
$$

Historically, this bound appears to be the first discrete analogue of the isoperimetric inequality (1.2). (A similar inequality also appears, in another context, in [De].) The Dodziuk bound (1.5) coupled with the uniform boundedness of the degree of a vertex from above ensures the transience of random walks on $G$. Bound (1.5) was significantly improved by Mohar [M4] who showed that

$$
h(\delta) \geq \frac{\delta-6}{\delta-4} \quad(\delta \geq 7) .
$$

The primary aim of this paper is to derive a still better lower bound for $h(\delta)$ :

$$
h(\delta) \geq \frac{(\delta-6)\left(\delta^{2}-8 \delta+15\right)}{(\delta-4)\left(\delta^{2}-8 \delta+13\right)} \quad(\delta \geq 7) .
$$

Note that bound (1.7) is for infinite, locally finite planar graphs with one end and minimum degree at least 7. The proof given in Sections 5 and 7 is combinatorial. In fact, bound (1.7) follows from our bounds for $h(G)$ when $G$ is a $\delta$-regular plane triangulation (Theorem 5.2) and when $G$ is a plane cell 2-complex with minimum degree $\delta$ (Theorem 7.1).

Recently, Häggström et al. [HJL] and, independently, Higuchi and Shirai [HS] have shown that in the special case when $G$ is a tessellation of the hyperbolic plane which is both $d$-vertex-regular and $f$-face-regular (with $1 / d+1 / f<\frac{1}{2}$ ), then

$$
h(G)=(f-2) \sqrt{1-\frac{4}{(d-2)(f-2)}} .
$$

In the case when the graph $G$ is both $d$-vertex-regular and $f$-face-regular, the value in (1.8) is better than the bound in (1.7). In fact, we believe that the value in (1.8) is the exact value for $h(\delta)$.

It is worth mentioning that Mohar [M4] studied infinite triangulations in a more general setting: he triangulated open subsets of surfaces with non-negative Euler characteristic, possibly with multiple ends. Most of his bounds are tight. However, in the case of infinite plane graphs with one end, his bound (1.6) is not tight and we improve his bound to that given in (1.7).

In Section 2 we obtain another lower bound for $h(G)$ where $G$ is a $\delta$-regular triangulation of the plane:

$$
h(G) \geq \frac{\pi(\delta-6)}{2 \delta} \cdot \frac{1}{\ln \left(D+\sqrt{D^{2}-1}\right)} \quad(\delta \geq 7),
$$

where $D=(2 \sin (\pi / \delta))^{-1}$. This lower bound is geometric; it is derived from (1.2) by a purely geometric argument. Although it is far from being tight, the method we use 
to obtain it demonstrates that the isoperimetric constant (1.3) is indeed an analogue of the Cheeger-Gromov-Yau constant. Surprisingly, we were unable to find any account of (1.9) in the literature. For $\delta=7$, this geometric bound is considerably tighter than the Mohar bound (1.6), but is weaker than our bound (1.7). However, as $\delta \rightarrow \infty$, the geometric bound (1.9) implies only the trivial inequality $h(\delta) \geq 0$.

In Section 3 we establish the following upper bound:

$$
h(\delta) \leq \sqrt{\frac{\delta-6}{\delta-2}} \quad(\delta \geq 7)
$$

The difference between (1.10) and our bound (1.7) is $O\left(\delta^{-6}\right)$, while the difference between (1.10) and the Mohar bound (1.6) is $O\left(\delta^{-2}\right)$ (as $\left.\delta \rightarrow \infty\right)$.

Conjecture 1.1. The upper bound (1.10) is the exact value for $h(\delta)(\delta \geq 7)$.

\section{The Geometric Lower Bound}

In this section we establish inequality (1.9). Let $G$ be a $\delta$-regular triangulation of the plane with $\delta \geq 7$. (Note that a $\delta$-regular triangulation of the plane with one end is uniquely determined.) We exploit the idea of Grünbaum and Shephard [GS] to realize $G$ geometrically, and refer the reader to [R] for foundations of hyperbolic geometry. We use the standard Poincaré model of the hyperbolic plane $\mathrm{H}^{2}$ with constant negative curvature $K=-1$. Draw an equilateral triangle $\Delta$ with angles of size $\alpha=2 \pi / \delta$, which has one of its vertices, $v_{0}$, in the center of the Poincare disk. Then tile the whole of $\mathrm{H}^{2}$ by repeatedly reflecting that triangle about its sides. The infinite tessellation of $\mathrm{H}^{2}$ thus obtained is a geometric realization of $G$. Remarkably, given a polygon $Q$ in $G$, the perimeter and area of $Q$ in $\mathrm{H}^{2}$ are multiples of the combinatorial quantities $l(\partial Q)=|E(\partial Q)|$ and $a(Q)=|F(Q)|$, respectively. More precisely, we need to multiply $l(\partial Q)$ by $L(\Delta)$, the length of the side of $\Delta$, to get the perimeter of $Q$ and multiply $a(Q)$ by $A(\Delta)$, the area of $\Delta$, to get the area of $Q$. It is a well known fact that $L(\Delta)$ and $A(\Delta)$ are functions of $\alpha$ and are determined from the following equations:

$$
\cosh \frac{L(\Delta)}{2} \cdot \sin \frac{\alpha}{2}=\frac{1}{2}, \quad A(\Delta)=\pi-3 \alpha \text {. }
$$

Now apply inequality (1.2) with $\varepsilon=1$ to the piecewise smooth curve $\partial Q$ to get (1.9).

Above we have used the standard model of $\mathrm{H}^{2}$ with $K=-1$, but even if we varied $K$, we could not do better than (1.9), using this geometric approach. We could assign to $K$ any negative constant value $-\varepsilon^{2}$, modifying (2.1) accordingly, but $\varepsilon$ would cancel out from the bound and we would obtain as the bound an expression involving only $\alpha$. However, $\alpha$ is determined to be $2 \pi / \delta$ by the $\delta$-regularity of $G$.

From the geometric construction it follows that $G$ has a so-called lens structure. More precisely, $G$ is the limit of the lens sequence $\left\{L_{n}\right\}_{n=0}^{\infty}$ centered at $v_{0}$, defined by recursion on $n$ as follows: $L_{0}=\left\{v_{0}\right\}$, and, for $n \geq 1, L_{n}$ is obtained from $L_{n-1}$ by adjoining the triangles of $G$ incident to $\partial L_{n-1}$ that do not lie in $L_{n-1}$. ( $L_{n}$ may be thought of as representing one side of an optical hyperbolic lens.) We chose $v_{0}$ as the center of 
the Poincaré disk. In fact, any vertex $v_{0} \in V(G)$ could be chosen as the center of a lens sequence. By a theorem of Grünbaum and Shephard [GS], the lens sequence is independent of the choice of the center $v_{0}$, up to homeomorphism of $\mathrm{H}^{2}$.

\section{The Upper Bound}

Suppose $\delta \geq 7$ and let $G$ be a $\delta$-regular triangulation of the plane, described geometrically in the preceding section. In this section we show that the bound (1.10) is approached from above by the lens sequence $\left\{L_{n}\right\}$.

It is convenient to describe $G$ also in graph-theoretic language. For a fixed vertex $v_{0} \in V(G)$, the vertices at distance $n$ from $v_{0}, n=0,1, \ldots$, determine a cycle $C_{n}=\partial L_{n}$. Thus $C_{n}$ are concentric cycles centered at $v_{0}$. All the edges of $G$ are either on $C_{n}$ or have one endvertex on $C_{n}$ and the other on $C_{n-1}$ or $C_{n+1}$, for some $n \geq 1$. Furthermore, each vertex $v \in V\left(C_{n}\right)$ is adjacent to one or two vertices on $C_{n-1}$, and is said to be of Type $I$ or $I I$ accordingly. Note that each Type I vertex on $C_{n}$ is thus adjacent to some $\delta-3$ vertices on $C_{n+1}$ and that each Type II vertex is adjacent to some $\delta-4$ vertices on $C_{n+1}$. Type II vertices occur as early as on $C_{2}$. Let $l_{n}$ denote $\left|E\left(C_{n}\right)\right|$, the length of $C_{n}$, and let $a_{n}$ denote $\left|F\left(L_{n}\right)\right|$.

The lemmas in this section are related to the $\delta$-regular $(\delta \geq 7)$ triangulation $G$ of the plane and assume the notation of the preceding paragraph.

Lemma 3.1. For the lens sequence $\left\{L_{n}\right\}$ we have

$$
l_{0}=1, \quad l_{n}=\frac{\delta}{\sqrt{(\delta-2)(\delta-6)}}\left(\alpha^{n}-\beta^{n}\right) \quad(n \geq 1),
$$

where

$$
\alpha=\frac{1}{2}(\delta-4+\sqrt{(\delta-2)(\delta-6)}), \quad \beta=\frac{1}{2}(\delta-4-\sqrt{(\delta-2)(\delta-6)}) .
$$

Proof. To write a recurrence relation for $l_{n}$, observe that $C_{n}$ (for $n \geq 2$ ) has $l_{n-1}$ vertices of degree 4 and $l_{n}-l_{n-1}$ vertices of degree 3 in $L_{n}$, so that

$$
l_{1}=\delta, \quad l_{2}=\delta(\delta-4), \quad l_{n}=(\delta-4) l_{n-1}-l_{n-2} \quad(n \geq 3) .
$$

Solving the characteristic equation of this recurrence relation, $x^{2}-(\delta-4) x+1=0$, we write the general solution,

$$
l_{n}=D_{1} \alpha^{n}+D_{2} \beta^{n} \quad(n \geq 1)
$$

and the values $D_{1}=-D_{2}=\delta / \sqrt{(\delta-6)(\delta-2)}$ are determined using the initial conditions.

Lemma 3.2. $\left\{l_{n} / l_{n-1}\right\}_{n \geq 1}$ is a strictly decreasing sequence. 
Proof. Since $\alpha$ and $\beta$ are solutions of the characteristic equation, we have $\alpha \beta=1$, and $\alpha-\beta=\sqrt{(\delta-2)(\delta-6)}$. Using Lemma 3.1 along with these identities, we obtain

$$
\begin{aligned}
l_{n}^{2}-l_{n-1} l_{n+1} & =\frac{\delta^{2}}{(\delta-2)(\delta-6)}\left[\left(\alpha^{n}-\beta^{n}\right)^{2}-\left(\alpha^{n-1}-\beta^{n-1}\right)\left(\alpha^{n+1}-\beta^{n+1}\right)\right] \\
& =\frac{\delta^{2}}{(\delta-2)(\delta-6)}\left(\alpha^{2}-2+\beta^{2}\right)=\frac{\delta^{2}}{(\delta-2)(\delta-6)}(\alpha-\beta)^{2}=\delta^{2} .
\end{aligned}
$$

Therefore the sequence $\left\{l_{n}\right\}_{n \geq 0}$ is, in fact, strictly log-concave down and the lemma follows.

Lemma 3.3. For the lens sequence $\left\{L_{n}\right\}$ the following two statements hold:

(1) $\left\{l_{n} / a_{n}\right\}_{n \geq 1}$ is a strictly decreasing sequence, and

(2) $\lim _{n \rightarrow \infty} l_{n} / a_{n}=\sqrt{(\delta-6) /(\delta-2)}$.

Proof. We prove part (1) first. Observe that $a_{k+1}-a_{k}=l_{k}+l_{k+1}$ for each $k=1,2, \ldots$. Hence,

$$
a_{n}=a_{1}+\sum_{k=1}^{n-1}\left(a_{k+1}-a_{k}\right)=l_{1}+\sum_{k=1}^{n-1}\left(l_{k}+l_{k+1}\right)=2\left(\sum_{k=1}^{n} l_{k}\right)-l_{n},
$$

and therefore

$$
\begin{aligned}
a_{n+1} l_{n}-a_{n} l_{n+1} & =\left[2\left(\sum_{k=1}^{n+1} l_{k}\right)-l_{n+1}\right] l_{n}-\left[2\left(\sum_{k=1}^{n} l_{k}\right)-l_{n}\right] l_{n+1} \\
& =2 l_{1} l_{n}+2 \sum_{k=2}^{n}\left(l_{k} l_{n}-l_{k-1} l_{n+1}\right) \\
& =2 l_{1} l_{n}+2 \sum_{k=2}^{n}\left(\frac{l_{k}}{l_{k-1}}-\frac{l_{n+1}}{l_{n}}\right) l_{k-1} l_{n} .
\end{aligned}
$$

By Lemma 3.2, $l_{k} / l_{k-1}-l_{n+1} / l_{n}>0$, since $k \leq n$. Hence $a_{n+1} l_{n}-a_{n} l_{n+1}>0$ and part (1) follows.

We proceed to prove part (2). From (3.3) we derive

$$
\frac{a_{n}}{l_{n}}=\frac{2}{l_{n}}\left(\sum_{k=1}^{n} l_{k}\right)-1 .
$$

Now, using (3.4), (3.2), the obvious double inequality $0<\beta<1<\alpha$, and routine algebraic manipulations with the second equation of (3.1), we obtain

$$
\lim _{n \rightarrow \infty} \frac{a_{n}}{l_{n}}=\frac{2 C_{1} \alpha}{1-\alpha}\left(\lim _{n \rightarrow \infty} \frac{1-\alpha^{n}}{C_{1} \alpha^{n}+C_{2} \beta^{n}}\right)-1=\frac{\alpha+1}{\alpha-1}=\sqrt{\frac{\delta-2}{\delta-6}} .
$$

The proof of part (2) is complete. 
Theorem 3.4. Bound (1.10) holds for $\delta \geq 7$.

Proof. By Lemma 3.3, parts (1) and (2), we have

$$
h(G) \leq \inf \frac{l_{n}}{a_{n}}=\sqrt{\frac{\delta-6}{\delta-2}}
$$

when $G$ is a $\delta$-regular plane triangulation, and the theorem follows.

\section{Reductions}

Lemma 4.1. Let $m_{i}, n_{i}(i=1,2)$, and $b$ be positive numbers. If $m_{1} / n_{1}>b$ and $m_{2} / n_{2} \geq b$, then $\left(m_{1}+m_{2}\right) /\left(n_{1}+n_{2}\right)>b$.

Proof. The proof is a straightforward verification.

Let $G$ be a plane cell 2-complex. For a plane subgraph $H$, "singular" vertices and edges are those where the carrier of $H$ fails to be a 2-manifold. More precisely, we define a singular vertex of $H$ as one of a small neighborhood of the carrier which, in $H$, is disconnected by the removal of $v$. A singular edge of $H$ is one not contained in any face of $H$.

Lemma 4.2. When determining $h(\delta)$ in (1.4) and $h(G)$ in (1.3) we may assume that $G$ is a triangulation and $H$ is a polygon, respectively, and therefore $l(\partial H)=|E(\partial H)|$ and $a(H)=|F(H)|$.

Proof. $G$ can be changed into a triangulation $G^{\prime}$ by appropriately triangulating the nontriangular faces (if any) without producing multiple edges. This procedure increases the value of the denominator in (1.3) and therefore a lower bound for $h\left(G^{\prime}\right)$ is a lower bound for $h(G)$.

By Lemma 4.1, we may assume that $H$ is connected. Next, we may assume that $H$ has no singular edge. For, suppose $H$ has a singular edge $e$. Let $H_{1}=H \backslash\{e\}$. Then $l\left(\partial H_{1}\right)=l(\partial H)-1, a(H)=a\left(H_{1}\right)$, and $l(\partial H) / a(H)>l\left(\partial H_{1}\right) / a\left(H_{1}\right)$. Hence a strict lower bound for $l\left(\partial H_{1}\right) / a\left(H_{1}\right)$ certainly is a strict lower bound for $l(\partial H) / a(H)$. Finally, if $H$ contains a singular vertex but no singular edge, then it can be easily seen that $H$ is a union of edge-disjoint polygons, possibly with holes. We then close all holes with new faces and note that this process only decreases the ratio (1.3) for each polygon with holes. Therefore we may assume that $H$ is a union of edge-disjoint polygons. Lemma 4.1 guarantees that the smallest among the ratios (1.3) for those polygons provides a lower bound on the ratio for the whole $H$. Therefore we may assume that $H$ is connected and contains no singular vertex and no holes, which means that $H$ is a single polygon. 


\section{The Lower Bound for Triangulations}

In this section we prove that the bound in (1.7) is a lower bound for $h(G)$ when $G$ is a $\delta$ regular plane triangulation. As noted in the Introduction, for $\delta$-regular triangulations, the result in (1.8) which appeared in the recent works [HJL] and [HS] is sharp and therefore better than the bound in (1.7). However, we use an approach different from that used by these authors because it subsequently extends to non-vertex-regular triangulations.

Suppose $u, v$ are two consecutive vertices on $C_{n}=\partial L_{n}$, where $u$ precedes $v$ in clockwise order. Denote by $u_{1}, \ldots, u_{\lambda}$ the neighbors of $u$, and by $v_{1}\left(=u_{\lambda}\right), \ldots, v_{\tau}$ the neighbors of $v$, on $C_{n+1}$ in clockwise order. The vertex $u_{1}$ ( $v_{1}$, respectively) is the first neighbor of $u(v)$, and $u_{\lambda}\left(v_{\tau}\right)$ is the last neighbor of $u(v)$. A forward section $R\left[u u_{\lambda} v_{\tau} v\right]$ with supporting edge $u v$ is defined to be the union of the triangle $u u_{\lambda} v$ and the triangles incident to $v$ which have an edge on $C_{n+1}$. Similarly, a backward section $R\left[u u_{1} u_{\lambda} v\right]$ is the union of the triangle $u u_{\lambda} v$ and the triangles incident to $u$ which have an edge on $C_{n+1}$. A forward section $R\left[u u_{\lambda} v_{\tau} v\right]$ is said to be of Type I (Type II, respectively) if $v$ is a Type I (Type II) vertex. Similarly, the Type (I or II) of a backward section $R\left[u u_{1} u_{\lambda} v\right]$ is determined by the Type of vertex $u$.

Let $A_{n}$ denote the closure of $L_{n+1} \backslash L_{n}$. A segment of $A_{n}$ is a polygon in $A_{n}$. Let $S$ be a segment of $A_{n}$. We use $U$ to represent a Type I section and $W$ to represent a Type II section. Then $A_{n}$ is a union of $U$ 's and $W$ 's:

$$
A_{n}=\cdots U U U W U \cdots U W U \cdots U W U U U \cdots .
$$

Note that there are never two consecutive Type II sections. If $U$ is a Type I section and $W$ is a Type II section, then

$\left|E\left(U \cap C_{n}\right)\right|=\left|E\left(W \cap C_{n}\right)\right|=1, \quad\left|E\left(U \cap C_{n+1}\right)\right|=\delta-4, \quad\left|E\left(W \cap C_{n+1}\right)\right|=\delta-5$.

Let $W_{1}$ and $W_{2}$ be two closest Type II forward (or both backward, respectively) sections in $A_{n}, n \geq 2$, with supporting edges $x_{1} y_{1}$ and $x_{2} y_{2}$, respectively. Then both $y_{1}$ and $y_{2}\left(x_{1}\right.$ and $\left.x_{2}\right)$ are Type II vertices, and $y_{1}\left(x_{1}\right)$ is the first neighbor of the apex vertex $u$ on $C_{n-1}$, and $y_{2}\left(x_{2}\right)$ is its last neighbor. (Figure 5.1 illustrates the situation in which $\delta=8$ and the shaded sections $W_{1}$ and $W_{2}$ are Type II forward sections.) If $u$ is a Type I vertex, there are $\delta-5$ Type I forward (backward) sections between $W_{1}$ and $W_{2}$. The union of $W_{1}\left(W_{2}\right)$ and those $\delta-5$ Type I sections is called a forward (backward) Type I subdivision supported by the path $P\left[x_{1} x_{2}\right]\left(P\left[y_{1}, y_{2}\right]\right)$. By $P[x y]$ we denote the path from vertex $x$ to vertex $y$ on $C_{n}$ taken in clockwise order. If $u$ is a Type II vertex, then a Type II subdivision is defined similarly, replacing " $\delta-6$ " with " $\delta-5$." $W_{1}\left(W_{2}\right)$ is called the leading section of the forward (backward) subdivision. If $\mathcal{U}$ is a Type I subdivision and $\mathcal{W}$ is a Type II subdivision, then

$$
\begin{aligned}
\left|E\left(\mathcal{U} \cap C_{n}\right)\right| & =\delta-4, \\
\left|E\left(\mathcal{U} \cap C_{n+1}\right)\right| & =(\delta-4)(\delta-5)+(\delta-5)=(\delta-3)(\delta-5), \\
\left|E\left(\mathcal{W} \cap C_{n}\right)\right| & =\delta-5, \\
\left|E\left(\mathcal{W} \cap C_{n+1}\right)\right| & =(\delta-4)(\delta-6)+(\delta-5)=\delta^{2}-9 \delta+19 .
\end{aligned}
$$




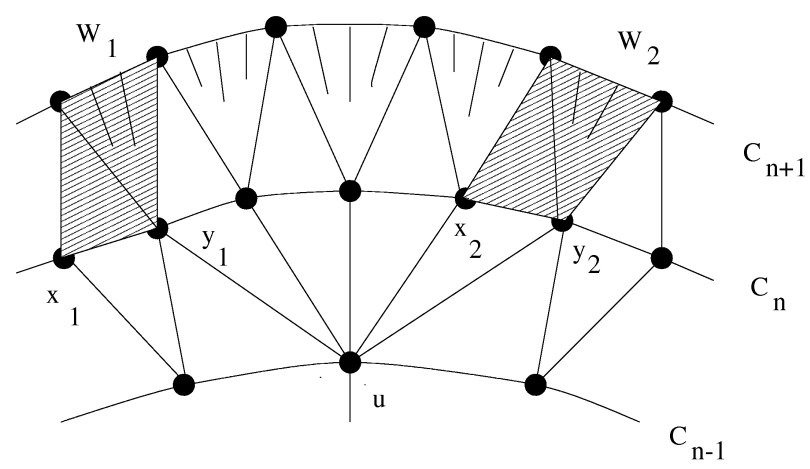

Fig. 5.1

Let $x, y, z$ be three consecutive vertices on $C_{n}$ with $y$ being a Type II vertex. Let $u$ be the last neighbor of $x$ (or, equivalently, the first neighbor of $y$ ) and let $v$ be the last neighbor of $y$ (or, equivalently, the first neighbor of $z$ ). The polygon bounded by the paths $P[x z]$ on $C_{n}$ and $P[u v]$ on $C_{n+1}$, and the edges $u x$ and $v z$ is called an $M$-section of $A_{n}$, denoted by $\mathcal{M}=\mathcal{M}[$ xuvz]. We have

$$
\left|E\left(\mathcal{M} \cap C_{n}\right)\right|=2, \quad\left|E\left(\mathcal{M} \cap C_{n+1}\right)\right|=\delta-5 .
$$

Let $Q$ be a polygon of $G$ and let $S$ be a segment in $Q \cap A_{n}$. Let $P\left[x_{1} x_{s}\right]$ be the path of $S \cap C_{n}$ and let $P\left[y_{1} y_{t}\right]$ be the path of $S \cap C_{n+1}$. If $y_{1}$ is the last neighbor of $x_{1}$ and $y_{t}$ is the first neighbor of $x_{s}$, and $x_{2}, x_{s-1}$ are both Type II vertices, then $S$ is called a minimal segment of $Q \cap A_{n}$. It is the union of some forward subdivisions and the M-section $\mathcal{M}\left[x_{s-2} y_{t-\delta+5} y_{t} x_{s}\right]$, where $y_{t-\delta+5}$ is the last neighbor of $x_{s-2}$. The minimal segment can also be viewed as the union of some backward subdivisions and the M-section $\mathcal{M}\left[x_{1} y_{1} y_{\delta-4} x_{3}\right]$, where $y_{\delta-4}$ is the first neighbor of $x_{3}$.

Lemma 5.1. Let $Q$ be a polygon of $G$ and let $\mathcal{S}$ be a minimal segment of $Q \cap A_{n}$. If $\mathcal{S}$ contains at most $\delta-5$ subdivisions, then

$$
\frac{\left|E\left(\mathcal{S} \cap C_{n+1}\right)\right|-\left|E\left(\mathcal{S} \cap C_{n}\right)\right|+2}{\left|E\left(\mathcal{S} \cap C_{n+1}\right)\right|+\left|E\left(\mathcal{S} \cap C_{n}\right)\right|}>\sqrt{\frac{\delta-6}{\delta-2}} .
$$

Proof. Denote the left side of inequality (5.1) by $g(\mathcal{S})$. If $x_{2}=x_{s-1}, \mathcal{S}$ is an M-section, so that $g(\mathcal{S})=(\delta-5) /(\delta-3)$. Then it is straightforward to verify (5.1). Now assume $x_{2} \neq x_{s-1}$ and there are $k(\geq 3)$ Type II vertices $x_{i_{1}}, \ldots, x_{i_{k}}$ in $P\left[x_{1} x_{s}\right]$ with $x_{i_{1}}=x_{2}$ and $x_{i_{k}}=x_{s-1}$. For each $i_{j}(j=1, \ldots, k-1), x_{i_{j}-1} x_{i_{j}}$ is the supporting edge of a forward Type II section, and each of these forward Type II sections is the leading section of a Type I or a Type II subdivision. Since $\mathcal{S}$ contains at most $\delta-5$ subdivisions, we have $k-1 \leq \delta-5$. Furthermore, since there are either $\delta-5$ or $\delta-6$ Type I subdivisions between two closest Type II subdivisions, $\mathcal{S}$ contains at most one Type II subdivision. 
Case 1: $\mathcal{S}$ is the union of $k-1$ forward Type I subdivisions and the M-section $\mathcal{M}\left[x_{s-2}\right.$ $\left.y_{t-\delta+5} y_{t} x_{s}\right]$. Then

$$
\begin{aligned}
g(\mathcal{S}) & =\frac{[(k-1)(\delta-3)(\delta-5)+(\delta-5)]-[(k-1)(\delta-4)+2]+2}{[(k-1)(\delta-3)(\delta-5)+(\delta-5)]+[(k-1)(\delta-4)+2]} \\
& =\frac{(k-1)\left(\delta^{2}-9 \delta+19\right)+\delta-5}{(k-1)\left(\delta^{2}-7 \delta+11\right)+\delta-3} .
\end{aligned}
$$

Denote this quantity by $g_{1}(\delta, k)$. Since

$$
\frac{\partial g_{1}(\delta, k)}{\partial k}=\frac{-2}{\left[(k-1)\left(\delta^{2}-7 \delta+11\right)+\delta-3\right]^{2}}<0,
$$

$g_{1}(\delta, k)$ is a decreasing function of $k$. Therefore to prove the lemma for Case 1, it suffices to show the inequality holds when $k=\delta-5$; that is, $g_{1}(\delta, \delta-5)>\sqrt{(\delta-6)(\delta-2)}$, which is a straightforward verification. Thus the lemma is true in this case.

Case 2: $\mathcal{S}$ is the union of the following: $k-2$ forward Type I subdivisions, one forward Type II subdivision, and the M-section $\mathcal{M}\left[x_{s-2} y_{t-\delta+5} y_{t} x_{s}\right]$. Then

$$
\begin{aligned}
g(\mathcal{S}) & =\frac{\left[(k-2)(\delta-3)(\delta-5)+\left(\delta^{2}-9 \delta+19\right)+(\delta-5)\right]}{\left[(k-2)(\delta-3)(\delta-5)+\left(\delta^{2}-9 \delta+19\right)+(\delta-5)\right]} \\
& =\frac{\delta^{2}-9 \delta+19}{\delta^{2}-7 \delta+11} .
\end{aligned}
$$

Then the inequality $g(\mathcal{S})>\sqrt{(\delta-6)(\delta-2)}$ is easily verified. This finishes the proof.

Theorem 5.2. Let $G$ be a $\delta$-regular triangulation of the plane with $\delta \geq 7$. Then

$$
h(G)>\frac{(\delta-6)\left(\delta^{2}-8 \delta+15\right)}{(\delta-4)\left(\delta^{2}-8 \delta+13\right)} .
$$

Proof. Denote the right side of (5.2) by $f(\delta)$. By Lemma 4.2, we only need to show $l(\partial Q) / a(Q)>f(\delta)$ for any polygon $Q$ in $G$.

Claim 1. If $\delta \geq 7, f(\delta)<\sqrt{(\delta-6)(\delta-2)}<1$.

Proof of Claim 1. The proof is a routine verification.

The proof of the theorem proceeds by induction on the number of triangles in $Q$. First we assume that $Q$ is a single triangle. Therefore $l(\partial Q) / a(Q)=3>1>f(\delta)$ and so the theorem holds in this case. Now assume that $Q$ is a polygon containing more than one triangle. If $Q$ contains no interior vertex, then $a(Q)=|E(\partial Q)|-2$. Since $|E(\partial Q)| /(|E(\partial Q)|-2)>1>f(\delta)$, the theorem also holds. Therefore we may assume 
that $Q$ contains an interior vertex, $v_{0}$. Let $\left\{L_{1}, \ldots, L_{n}, \ldots\right\}$ be the lens sequence centered at $v_{0}$. Let $n$ be the largest index such that $Q \cap A_{n} \neq \emptyset$. Let $\mathcal{S}$ be a segment of $Q \cap A_{n}$ with $\mathcal{S} \cap C_{n}=P\left[x_{1} x_{s}\right]$ and $\mathcal{S} \cap C_{n+1}=P\left[y_{1} y_{t}\right]$.

Case 1: $y_{t}$ is not the first neighbor of $x_{s}$. Let $y_{t}^{\prime}$ be the first neighbor of $x_{s}$. Let $Q^{-}$be the polygon obtained from $Q$ by deleting the triangles incident to $x_{s}$ which have an edge on $C_{n+1}$. Suppose $k$ triangles are deleted. Then $l(\partial Q) / a(Q)=\left(l\left(\partial Q^{-}\right)+k\right) /\left(a\left(Q^{-}\right)+k\right)$. By the induction hypothesis, $l\left(\partial Q^{-}\right) / a\left(Q^{-}\right)>f(\delta)$. Since $k / k=1>f(\delta)$, then $l(\partial Q) / a(Q)>f(\delta)$, by Lemma 4.1. Therefore the theorem holds in this case.

Case 2: $y_{t}$ is the first neighbor of $x_{s}$, and $x_{s-1}$ is a Type I vertex. Then the edge $x_{s-1} x_{s}$ is the supporting edge of a backward Type I section $U$. Let $Q^{-}=Q \backslash U$. Then

$$
\frac{l(\partial Q)}{a(Q)}=\frac{l\left(\partial Q^{-}\right)+\left|E\left(U \cap C_{n+1}\right)\right|-\left|E\left(U \cap C_{n}\right)\right|}{a\left(Q^{-}\right)+\left|E\left(U \cap C_{n+1}\right)\right|+\left|E\left(U \cap C_{n}\right)\right|}=\frac{l\left(\partial Q^{-}\right)+(\delta-4)-1}{a\left(Q^{-}\right)+(\delta-4)+1} .
$$

By the induction hypothesis, $l\left(\partial Q^{-}\right) / a\left(Q^{-}\right)>f(\delta)$. Since $(\delta-5) /(\delta-3)>f(\delta)$, then $l(\partial Q) / a(Q)>f(\delta)$, by Lemma 4.1 .

Case 3: $y_{t}$ is the first neighbor of $x_{s}$, and $x_{s-1}$ is a Type II vertex.

Subcase 3.1: $\mathcal{S}$ contains at least $\delta-5$ subdivisions (Type I or Type I and Type II combined). Then the edge $x_{s-1} x_{s}$ is the supporting edge of a backward Type II section. Denote this section by $W_{1}$. If to the left of $W_{1}$ there are $\delta-5$ Type I sections, then $W_{1}$ and these $\delta-5$ Type I sections together form a Type I subdivision. Denote this subdivision by $\mathcal{U}$. Let $Q^{-}=Q \backslash \mathcal{U}$. Recalling the formulas for $\left|E\left(\mathcal{U} \cap C_{n}\right)\right|$ and $\left|E\left(\mathcal{U} \cap C_{n+1}\right)\right|$, we obtain

$$
\begin{aligned}
\frac{l(\partial Q)}{a(Q)} & =\frac{l\left(\partial Q^{-}\right)+\left|E\left(\mathcal{U} \cap C_{n+1}\right)\right|-\left|E\left(\mathcal{U} \cap C_{n}\right)\right|}{a\left(Q^{-}\right)+\left|E\left(\mathcal{U} \cap C_{n+1}\right)\right|+\left|E\left(\mathcal{U} \cap C_{n}\right)\right|} \\
& =\frac{l\left(\partial Q^{-}\right)+(\delta-5)(\delta-3)-(\delta-4)}{a\left(Q^{-}\right)+(\delta-5)(\delta-3)+(\delta-4)} .
\end{aligned}
$$

By the induction hypothesis, $l\left(\partial Q^{-}\right) / a\left(Q^{-}\right)>f(\delta)$. Since

$$
\frac{(\delta-5)(\delta-3)-(\delta-4)}{(\delta-5)(\delta-3)+(\delta-4)}>f(\delta),
$$

then $l(\partial Q) / a(Q)>f(\delta)$, by Lemma 4.1. If to the left of $W_{1}$, there are only $\delta-6$ consecutive Type I sections, then $W_{1}$ and these $\delta-6$ Type I sections together form a Type II subdivision which we denote by $\mathcal{W}$. Since between two closest Type II subdivisions there are either $\delta-5$ or $\delta-6$ Type I subdivisions, then to the left of $\mathcal{W}$ there are at least $\delta-6$ consecutive Type I subdivisions $\mathcal{U}_{1}, \ldots, \mathcal{U}_{\delta-6}$. Let $Q^{\prime}=\mathcal{W} \cup \mathcal{U}_{1} \cup \cdots \cup \mathcal{U}_{\delta-6}$. As above, $\left|E\left(Q^{\prime} \cap C_{n}\right)\right|=(\delta-6)(\delta-4)+(\delta-5)$ and $\left|E\left(Q^{\prime} \cap C_{n+1}\right)\right|=(\delta-6)(\delta-3)(\delta-$ $5)+\left(\delta^{2}-9 \delta+19\right)$. Let $Q^{-}=Q \backslash L^{\prime}$. Then

$$
\frac{l(\partial Q)}{a(Q)}=\frac{l\left(\partial Q^{-}\right)+\left|E\left(Q^{\prime} \cap C_{n+1}\right)\right|-\left|E\left(Q^{\prime} \cap C_{n}\right)\right|}{a\left(Q^{-}\right)+\left|E\left(Q^{\prime} \cap C_{n+1}\right)\right|+\left|E\left(Q^{\prime} \cap C_{n}\right)\right|} .
$$


By the induction hypothesis, $l\left(\partial Q^{-}\right) / a\left(Q^{-}\right)>f(\delta)$. Since

$$
\begin{aligned}
& \frac{\left|E\left(Q^{\prime} \cap C_{n+1}\right)\right|-\left|E\left(Q^{\prime} \cap C_{n}\right)\right|}{\left|E\left(Q^{\prime} \cap C_{n+1}\right)\right|+\left|E\left(Q^{\prime} \cap C_{n}\right)\right|} \\
& \quad=\frac{\left((\delta-6)(\delta-3)(\delta-5)+\left(\delta^{2}-9 \delta+19\right)\right)-((\delta-6)(\delta-4)+(\delta-5))}{\left((\delta-6)(\delta-3)(\delta-5)+\left(\delta^{2}-9 \delta+19\right)\right)+((\delta-6)(\delta-4)+(\delta-5))} \\
& \quad=f(\delta),
\end{aligned}
$$

then $l(\partial Q) / a(Q)>f(\delta)$, by Lemma 4.1.

Subcase 3.2: $\mathcal{S}$ contains less than $\delta-5$ subdivisions (Type I or Type I and Type II combined). By the discussion in Cases 1 and 2, and the symmetry, we may assume that $x_{2}$ is a Type II vertex. Therefore $S$ is a minimal segment. Let $Q^{-}=Q \backslash \mathcal{S}$. Then

$$
\frac{l(\partial Q)}{a(Q)}=\frac{l\left(\partial Q^{-}\right)+\left|E\left(\mathcal{S} \cap C_{n+1}\right)\right|-\left|E\left(S \cap C_{n}\right)\right|+2}{a\left(Q^{-}\right)+\left|E\left(\mathcal{S} \cap C_{n+1}\right)\right|+\left|E\left(S \cap C_{n}\right)\right|} .
$$

By the induction hypothesis, $l\left(\partial Q^{-}\right) / a\left(Q^{-}\right)>f(\delta)$. By Lemma 5.1 and Claim 1,

$$
\frac{l(\partial Q)}{a(Q)}=\frac{\left|E\left(\mathcal{S} \cap C_{n+1}\right)\right|-\left|E\left(S \cap C_{n}\right)\right|+2}{\left|E\left(\mathcal{S} \cap C_{n+1}\right)\right|+\left|E\left(S \cap C_{n}\right)\right|}>\sqrt{\frac{\delta-6}{\delta-2}}>f(\delta) .
$$

Then, by Lemma 4.1, $l(\partial Q) / a(Q)>f(\delta)$. This finishes the proof.

\section{A Lens Structure Theorem}

In this section we prove that infinite locally finite plane graphs with one end and with minimum degree $\delta \geq 6$ have a lens structure.

Lemma 6.1 [PZ]. Suppose finite plane graph $G$ has its infinite face bounded by a cycle $C$ and that $u$ and $v$ are two vertices on $C$. If the vertices on $C$, except possibly one or two vertices, have degree at least 4 , then there is an interior vertex with degree less than 6.

Lemma 6.2. Any infinite locally finite plane graph $G$ with one end and with minimum degree $\delta \geq 6$ is 3-connected.

Proof. Suppose to the contrary that $G$ is not 3-connected. Then there exists a 1-vertex cut or a 2-vertex cut. On one side of the cut there is a finite subgraph which satisfies the conditions of Lemma 6.1, and a contradiction is immediate.

The definition of a lens sequence for a general plane graph $G$ is obtained from the one given in Section 2 for triangulations by replacing "triangles" with "faces."

Theorem 6.3 (Lens Structure Theorem). Suppose $G$ is an infinite locally finite plane graph with one end and with minimum degree at least 6 . Let $\left\{L_{n}\right\}$ be the lens sequence 
centered at a vertex $v_{0}$. Then, for $n \geq 1$ :

(i) $L_{n}$ is a 2-disk, $\partial L_{n}$ is a cycle $C_{n}$, and $C_{n}$ is vertex-disjoint from $C_{n-1}$. Hence $C_{1}, C_{2}, \ldots$ together form a collection of concentric cycles.

(ii) Each edge in $L_{n} \backslash L_{n-1}$ is either contained in the cycle $C_{n}$, or has one endvertex on $C_{n-1}$ and the other on $C_{n}$.

(iii) Each vertex on $C_{n}$ is incident to at most two vertices on $C_{n-1}$.

Proof. This proof proceeds by induction on $n, n \geq 1$. By Lemma 6.2, $G$ is 3-connected, and so $L_{1}$ is a 2-disk. Now assume the theorem is true for all $k \leq n$. Therefore $L_{n}$ is a 2-disk bounded by the cycle $C_{n}$ and contains $n$ nested cycles $C_{1}, \ldots, C_{n}$ centered at $v_{0}$. Furthermore, the edges of $L_{n}$ either lie in these cycles or join a vertex in $C_{j}$ to a vertex in $C_{j+1}$, for some $0 \leq j \leq n-1$. We proceed to show that the theorem is also true for $k=n+1$. Let $x_{1}, \ldots, x_{m}$ denote the vertices on $C_{n}$ in clockwise order. Since $x_{1} \in \partial L_{n}$, some faces of st $\left(x_{1}\right)$ are in $L_{n}$. By the lens structure of $L_{n}$, the faces of st $\left(x_{1}\right)$ contained in $L_{n}$ together form a "half star." Let st $\left(x_{1}\right)^{-}=\operatorname{st}\left(x_{1}\right) \backslash L_{n}$. Then st $\left(x_{1}\right)^{-}$is the half star of the faces which are not in $L_{n}$. Clearly, the 2-edge path $x_{m} x_{1} x_{2}$ is a component of $\partial \operatorname{st}\left(x_{1}\right)^{-} \cap \partial L_{n}$.

Claim 2. This 2-edge path is the only component of $\partial s t\left(x_{1}\right)^{-} \cap \partial L_{n}$.

Proof of Claim 2. Suppose $\partial \mathrm{st}\left(x_{1}\right)^{-} \cap \partial L_{n}$ has a second component; in other words, suppose one of the faces incident to $x_{1}$ and contained in st $\left(x_{1}\right)^{-}$has a vertex $x_{k}$ on its boundary, for some $x_{k} \in C_{n}, k>2$. If $\partial \operatorname{st}\left(x_{1}\right)^{-} \cap \partial L_{n}$ has more than two components, choose $k$ as small as possible. Denote this face by $f$. (See Fig. 6.1.) Let $H$ be the subgraph of $G$ bounded by the path $P\left[x_{1} x_{k}\right]$ (or $P\left[x_{2} x_{k}\right]$ if $x_{2}$ is also on the boundary of $f$ ) and the section of $\partial f$ from $x_{1}\left(x_{2}\right)$ to $x_{k}$ in counterclockwise order. By the induction hypothesis, any vertex on $P\left[x_{1} x_{k}\right]$ is adjacent to at most two vertices on $C_{n-1}$, and therefore the degree of each vertex on $P\left[x_{1} x_{k}\right]$ (not including $x_{1}$ and $x_{k}$, or $x_{2}$ and $x_{k}$ ) in $H$ is at least 4, and the remaining vertices other than $x_{1}\left(x_{2}\right)$ and $x_{k}$ have degree at least 6 in $H$.

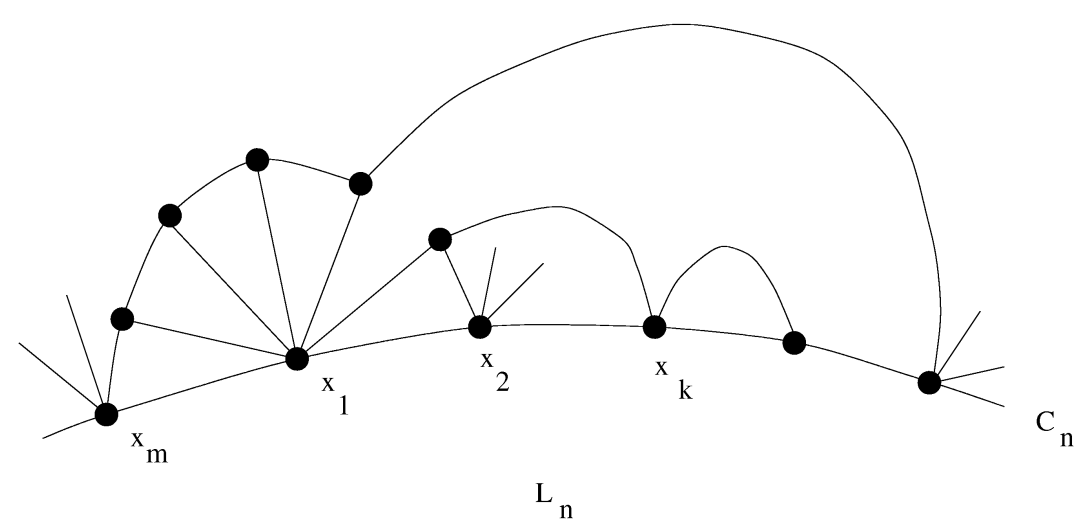

Fig. 6.1 
However, by Lemma 6.1, such a graph $H$ cannot exist. Hence, $\partial f \cap \partial L_{n}$ has no second component, and Claim 2 follows.

Now let $L_{n}\left[x_{1}\right]=\operatorname{st}\left(x_{1}\right)^{-} \cup L_{n}$. By Claim 2, $L_{n}\left[x_{1}\right]$ is a 2-disk. $\partial L_{n}\left[x_{1}\right]$ is the union of the path of $C_{n}$ from $x_{2}$ to $x_{m}$ and the path of $\partial \operatorname{st}\left(x_{1}\right)$ from $x_{m}$ to $x_{2}$ (clockwise).

Let $\operatorname{st}\left(x_{2}\right)^{-}=\operatorname{st}\left(x_{2}\right) \backslash L_{n}\left[x_{1}\right]$ and $L_{n}\left[x_{1}, x_{2}\right]=L_{n}\left[x_{1}\right] \cup \operatorname{st}\left(x_{2}\right)^{-}$.

Claim 3. $\partial \operatorname{st}\left(x_{2}\right)^{-} \cap \partial L_{n}\left[x_{1}\right]$ consists of a single component.

Proof of Claim 3. This proof is similar to that of Claim 2. Clearly, $\partial \operatorname{st}\left(x_{2}\right)^{-} \cap \partial L_{n}\left[x_{1}\right]$ has a component which is the union of the edge $x_{2} x_{3}$ and the path of $\partial \operatorname{st}\left(x_{1}\right)^{-}$from a vertex $w$ to $x_{2}$ (clockwise about $x_{1}$ ). Suppose there is a face $f$ in $\operatorname{st}\left(x_{2}\right)^{-}$which has a second attachment with $\partial L_{n}\left[x_{1}\right]$. If this second attachment is on the section of $C_{n}$ from $x_{3}$ to some $x_{l}$, then, by an argument similar to that used for Claim 2, such an attachment does not exist. If the second attachment is on the section of $\operatorname{st}\left(x_{1}\right)^{-}$from $x_{m}$ to $w$ (clockwise about $x_{1}$ ), then let $y$ be the vertex of this attachment closest to $w$ clockwise on $\partial \operatorname{st}\left(x_{i}\right)^{-}$. Let $H$ be the subgraph bounded by the section of $\partial \operatorname{st}\left(x_{1}\right)^{-}$from $y$ to $x_{2}$ (clockwise) and the section of $\partial f$ from $y$ to $x_{2}$ (counterclockwise with respect to $f$ ). All the vertices of $H$ on the section of $\partial \operatorname{st}\left(x_{1}\right)^{-}$from $y$ to $x_{2}$ (not including $y$ and $x_{2}$ ) have degree at least 5 in $H$, and thus satisfy the condition of Lemma 6.1. Therefore, by Lemma 6.1, $H$ does not exist, and Claim 3 follows.

Let $\operatorname{st}\left(x_{l}\right)^{-}=\operatorname{st}\left(x_{l}\right) \backslash L_{n}\left[x_{1}, \ldots, x_{l-1}\right]$ and $L_{n}\left[x_{1}, \ldots, x_{l}\right]=L_{n}\left[x_{1}, \ldots, x_{l-1}\right] \cup$ $\operatorname{st}\left(x_{l}\right)^{-}$, for $2 \leq l<m$. Assume $L_{n}\left[x_{1}, \ldots, x_{t}\right]=L_{n}\left[x_{1}, \ldots, x_{t-1}\right] \cup \operatorname{st}\left(x_{t}\right)$ is a 2-disk, for $t=2, \ldots, l$. We now show that $L_{n}\left[x_{1}, \ldots, x_{l+1}\right]=L_{n}\left[x_{1}, \ldots, x_{l}\right] \cup$ $\operatorname{st}\left(x_{l+1}\right)^{-}$is a 2-disk, where $\operatorname{st}\left(x_{l+1}\right)^{-}=\operatorname{st}\left(x_{l+1}\right) \backslash L_{n}\left[x_{1}, \ldots, x_{l}\right]$. It suffices to show that $\partial L_{n}\left[x_{1}, \ldots, x_{l}\right] \cap \partial \operatorname{st}\left(x_{l+1}\right)^{-}$consists of a single component. Let $w$ be the vertex on $\partial L_{n}\left[x_{1}, \ldots, x_{l+1}\right]$ preceding $x_{l}$ (clockwise about $x_{l+1}$ ). Then the 2-edge path $w x_{l+1} x_{l+2}$ is a component of $\partial L_{n}\left[x_{1}, \ldots, x_{l}\right] \cap \partial \operatorname{st}\left(x_{l+1}\right)^{-}$. Suppose there is a face $f$ in $\operatorname{st}\left(x_{l+1}\right)^{-}$ which has a second attachment with $\partial L_{n}\left[x_{1}, \ldots, x_{l}\right]$. If this second attachment is on the section of $C_{n}$ from $x_{l+2}$ to $x_{m}$, then by an argument similar to that used in Claim 2 , such an attachment does not exist. If the second attachment is on $\partial L_{n}\left[x_{1}, \ldots, x_{l}\right]$, then by an argument similar to that used in Claim 3, such an attachment does not exist. Therefore $\partial L_{n}\left[x_{1}, \ldots, x_{l}\right] \cap \partial \operatorname{st}\left(x_{l+1}\right)^{-}$consists of a single component which is a 2-edge path. Hence, $L_{n}\left[x_{1}, \ldots, x_{l+1}\right]=L_{n}\left[x_{1}, \ldots, x_{l}\right] \cup \operatorname{st}\left(x_{l+1}\right)^{-}$is a 2-disk. This inductive process constructively proves that $L_{n+1}=L_{n}\left[x_{1}, \ldots, x_{m}\right]$ is a 2-disk; denote by $C_{n+1}$ its boundary cycle. Since $C_{n+1}$ is the symmetric difference of $C_{n}$ and the boundaries of the faces incident to $C_{n}$, it follows that $C_{n} \cap C_{n+1}=\emptyset$. This proves (i).

Since $L_{n+1}=L_{n}\left[x_{1}, \ldots, x_{m}\right]$, each edge of $L_{n+1} \backslash L_{n}$ is either a rim edge or a spoke in some $\operatorname{st}\left(x_{j}\right)^{-}$. Furthermore, the rim edges are in $\partial L_{n+1}=C_{n+1}$ while the spokes join a vertex in $C_{n+1}$ to a vertex in $C_{n}$. Hence (ii) is true.

Suppose that there is a vertex on $C_{n+1}$ adjacent to more than two vertices on $C_{n}$. Let $v_{a}, v_{b}$, and $v_{c}$ be three consecutive such vertices on $C_{n}$ in clockwise order on $C_{n}$. By the induction hypothesis, there are at most two edges joining $v_{b}$ to the vertices on $C_{n-1}$. This implies that $v_{b}$ has degree at most 5 , a contradiction. Hence, (iii) is also true. 
Corollary 6.4. The sets $V(G), E(G)$, and $F(G)$ of any infinite locally finite plane graph $G$ with one end and minimum degree at least 6 give rise to a plane cell 2-complex.

Theorem 6.3 does not hold if we relax the restriction on $\delta$ to read $\delta \geq 5$. For, choose several faces and insert a subgraph with minimum degree 5 into each. Then the lens $L_{n}$ is not necessarily a 2-disk and $\partial L_{n}$ may be a union of two or more cycles.

\section{The Lower Bound in a General Setting}

In this section we show that the lower bound in Theorem 5.2 holds in the more general case in which the plane graphs considered are not necessarily regular and are not even required to be triangulations.

Theorem 7.1. Inequality (5.2) holds for any plane cell 2-complex $G$ with minimum degree at least 7.

Proof. First recall that, by Lemma 4.2, we may assume that $G$ is a plane triangulation and we only need to show that (5.2) holds for any polygon $Q$ of $G$. If $Q$ consists of a single triangle, $l(\partial Q) / a(Q)=\frac{3}{1}>f(\delta)$, and the theorem is true. If $Q$ contains more than one triangle but no interior vertex, $a(Q)=|E(\partial Q)|-2$, so that $l(\partial Q) / a(Q)>1>f(\delta)$, and so the theorem holds. Now we assume that $Q$ has an interior vertex, which we denote by $v_{0}$. By Theorem 6.3, $G$ has a lens structure, centered at $v_{0}$. Let $n$ be the largest index such that $Q \cap A_{n} \neq \emptyset$ and let $\mathcal{S}$ be a segment of $Q \cap A_{n}$. Let $\mathcal{S} \cap C_{n}=P\left[x_{1} x_{S}\right]$ and let $\mathcal{S} \cap C_{n+1}=P\left[y_{1} y_{t}\right]$.

Even if $G$ is not regular, each Type I (Type II, respectively) vertex $v$ on $C_{n}$ is still adjacent to one vertex (two vertices) on $C_{n-1}$. However, $v$ may be adjacent to more than $\delta-3\left(\delta-4\right.$, respectively) vertices on $C_{n+1}$, and so most of the counting formulas in Section 5 for the numbers of edges no longer hold.

Claim 4. We may assume that $\mathcal{S}$ is a segment $\mathcal{S}^{\prime}$ of $Q^{\prime} \cap A_{n}^{\prime}$, where $Q^{\prime}$ is a polygon in some triangulation $G^{\prime}$, centered at $v_{0}$, which is $\delta$-regular, and $A_{n}^{\prime}=L_{n+1}^{\prime} \backslash L_{n}^{\prime}$.

Proof of Claim 4. For each vertex $v$ on $C_{n} \cap \mathcal{S}$ with degree greater than $\delta$, contract $\operatorname{deg}_{G}(v)-\delta$ consecutive rim edges on $\operatorname{st}(v) \cap C_{n+1}$ into a single vertex with collapsing the incident faces into a single edge. This process transforms $\mathcal{S}$ to $\mathcal{S}^{\prime}$. Since

$$
\frac{l(\partial Q)}{a(Q)}=\frac{l\left(\partial Q^{\prime}\right)+\operatorname{deg}_{G}(v)-\delta}{a\left(Q^{\prime}\right)+\operatorname{deg}_{G}(v)-\delta} \quad \text { and } \quad \frac{\operatorname{deg}_{G}(v)-\delta}{\operatorname{deg}_{G}(v)-\delta}=1>f(\delta),
$$

it suffices to show that inequality (5.2) holds for $Q^{\prime}$ in $G^{\prime}$, by Lemma 4.1. In other words, we may suppose that all the vertices on $C_{n}$ have degree equal to $\delta$.

Next, suppose $x_{a}$ and $x_{b}$ are two closest Type II vertices on $P\left[x_{1} x_{s}\right]$. Then $P\left[x_{a} x_{b}\right]$ is the supporting path of a subdivision $\mathcal{T}$. Note that if $G$ is $\delta$-regular, between two closest Type II vertices on $C_{n}$ there are either $\delta-5$ Type I vertices (corresponding to a Type I subdivision) or $\delta-6$ Type I vertices (corresponding to a Type II subdivision). Let $u$ 
be the apex vertex of $\mathcal{T}$. If $u$ is a Type I vertex (hence $\mathcal{U}$ is a Type I subdivision) and $P\left[x_{a} x_{b}\right]$ contains $k+(\delta-5)$ Type I vertices, $k \geq 1$, then let $x_{i_{1}}, \ldots, x_{i_{k}}$ be the first $k$ Type I vertices and let $U_{1}, \ldots, U_{k}$ be the corresponding Type I sections supported by the edges $x_{a} x_{i_{1}}, \ldots, x_{i_{k-1}} x_{i_{k}}$, respectively. Let $\mathcal{T}^{\prime}=U_{1} \cup \cdots \cup U_{k}$. Contract the edges of $\mathcal{T}^{\prime} \cap\left(C_{n} \cup C_{n+1}\right)$ to shrink $\mathcal{T}^{\prime}$ to a single edge joining $C_{n}$ to $C_{n+1}$. Suppose this process transforms $Q$ to $Q^{\prime}$. Then

$$
\frac{l(\partial Q)}{a(Q)}=\frac{l\left(\partial Q^{\prime}\right)+k(\delta-4)}{a\left(Q^{\prime}\right)+k(\delta-4)+k} .
$$

Since $k(\delta-4) /(k(\delta-4)+k)=(\delta-4) /(\delta-3)>f(\delta)$, it suffices to show that $l\left(\partial Q^{\prime}\right) / a\left(Q^{\prime}\right)>f(\delta)$. Therefore we may assume that if $u$ is a Type I vertex, then the subdivision $\mathcal{T}$ consists of exactly one Type II section and $\delta-5$ Type I sections, which is the same as a Type I subdivision in a $\delta$-regular triangulation. Similarly, if $u$ is a Type II vertex, we may assume that the subdivision $\mathcal{T}$ consists of exactly one Type II section and $\delta-6$ Type I sections, just as a Type II subdivision in a $\delta$-regular triangulation does. This finishes the proof of Claim 4.

Now use Claim 4 and proceed as in the proof of Theorem 5.2 to finish the proof.

\section{Acknowledgments}

The authors thank John Ratcliffe and Mark Ellingham for useful discussions and the referees for their informative and constructive comments.

\section{References}

[BMST] N. L. Biggs, B. Mohar, and J. Shawe-Taylor, The spectral radius of infinite graphs, Bull. London Math. Soc. 20 (1988), 116-120.

[C] J. Cheeger, A lower bound for the smallest eigenvalue of the Laplacian, in Problems in Analysis, A Symposium in Honor of S. Bochner, pp. 195-199, Princeton University Press, Princeton, NJ, 1970.

[De] D. R. DeBaun, $L_{2}$-cohomology of noncompact surfaces, Trans. Amer. Math. Soc. 284 (1984), $543-$ 565.

[Do] J. Dodziuk, Difference equations, isoperimetric inequality and transience of certain random walks, Trans. Amer. Math. Soc. 284 (1984), 787-794.

[G] M. Gromov, Hyperbolic groups, in Essays in Group Theory, pp. 75-263, Springer-Verlag, New York, 1987.

[GS] B. Grünbaum and G. C. Shephard, Edge-transitive planar graphs, J. Graph Theory 11 (1987), 141155 .

[HJL] O. Häggström, J. Jonasson, and R. Lyons, Explicit isoperimetric constants and phase transitions in the random-cluster model, Ann. Probab. 30 (2002), 443-473.

[HS] Y. Higuchi and T. Shirai, Isoperimetric constants of $(d, f)$-regular planar graphs, preprint, June 2000.

[M1] B. Mohar, Isoperimetric inequalities, growth and the spectrum of graphs, Linear Algebra Appl. 103 (1988), 119-131.

[M2] B. Mohar, Embeddings of infinite graphs, J. Combin. Theory Ser. B 44 (1988), 29-43.

[M3] B. Mohar, Isoperimetric numbers of graphs, J. Combin. Theory Ser. B 47 (1989), 274-291.

[M4] B. Mohar, Isoperimetric numbers and spectral radius of some infinite planar graphs, Math. Slovaca 42 (1992), 411-425. 
[MW] B. Mohar and W. Woess, A survey on spectra of infinite graphs, Bull. London Math. Soc. 21 (1989), 209-234.

[PZ] M. D. Plummer and X. Zha, Genus bounds for embeddings with large connectivity and representativity, Discrete Math. 249 (2002), 167-178.

[R] J. G. Ratcliffe, Foundations of Hyperbolic Manifolds, Springer-Verlag, New York, 1994.

[S] P. M. Soardi, Recurrence and transience of the edge graph of a tiling of the Euclidean plane, Math. Ann. 287 (1990), 613-626.

[W] W. Woess, A note on tilings and strong isoperimetric inequality, Math. Proc. Cambridge Philos. Soc. 124 (1998), 385-393.

[Y] S. T. Yau, Isoperimetric constants and the first eigenvalue of a compact Riemannian manifold, Ann. Sci. École Norm. Sup. (4) 8 (1975), 487-507.

[Z] A. Zuk, On the norms of the random walks on planar graphs, Ann. Inst. Fourier (Grenoble) 47 (1997), 1463-1490.

Received August 15, 2000, and in revised form September 5, 2001, and May 16, 2002.

Online publication August 12, 2002. 